\title{
Calmodulin distribution in peripheral nerve: an EM immunocytochemical study
}

\author{
Marina Mata and David J. Fink \\ Neurology Research Laboratory, University of Michigan and VA Medical Center, Ann Arbor, MI 48105 (U.S.A.)
}

(Accepted 14 June 1988)

Key words: Calmodulin; Peripheral nerve; Axon; Schwann cell; Immunocytochemistry; Schmidt-Lanterman cleft

\begin{abstract}
We used electron microscopic immunocytochemistry to study the distribution of calmodulin in rat sciatic nerve. Calmodulin immunoreactivity was found throughout the axoplasmic matrix, but particularly along microtubules. Schwann cell cytoplasm and nuclei demonstrated immunoreactivity, while compact myelin did not. There was particularly intense immuno-gold deposition within Schmidt Lanterman clefts. At the nodes of Ranvier, calmodulin appeared preferentially in the paranodal region, along the apposition of the axolemma to the paranodal loops of myelin and extending into the paranodal loops. The presence of calmodulin immunoreactivity along microtubules supports biochemical and pharmacological evidence of calmodulin involvement in regulating the assembly and phosphorylation of microtubules, and in fast axonal transport along microtubules. The co-localization of paranodal calmodulin immunoreactivity with Ca-ATPase activity demonstrated cytochemically (Mata et al., Brain Reseach, in press) supports the notion that the paranodal Ca-ATPase activity may be regulated by calmodulin, and agrees with the in vitro biochemical evidence for Ca-ATPase of other cells.
\end{abstract}

\section{INTRODUCTION}

Calmodulin is an intracellular calcium binding protein which serves as the regulator for a variety of processes, transducing the effect of a slight rise in intracellular cytoplasmic calcium to create specific physiologic effects. To define the functional role of calmodulin in a cell requires that in vitro demonstration of calmodulin-mediated regulation be combined with appropriate ultrastructural localization of the regulating protein to account for its proposed role ${ }^{17}$.

In nervous tissue calmodulin-mediated regulation has been demonstrated in vitro for enzymes including cyclic nucleotide phosphodiesterase ${ }^{5}$, adenylate cyclase $^{3}$, cGMP protein kinase ${ }^{31}$, phosphoprotein phosphatase $^{29}$ and Ca,Mg-ATPase ${ }^{23}$. Calmodulin involvement in the dynamic regulation of the actinmyosin microfilaments through regulation of myosin light-chain kinase ${ }^{20}$, and in the regulation of microtubule polymerization-depolymerization equilibri$\mathrm{um}^{1.19}$ suggests that it may also play a role in the regulation of the neuronal cytoskeleton.
Immunocytochemical studies of calmodulin localization in central nervous system have yielded conflicting results. At the light microscopic level $\mathrm{CaM}$ is seen in neurons, and lesser amounts of immunoreactivity have been described in glial cells ${ }^{4,21,26,30}$. At the ultrastructural level, reports have included descriptions of immunostaining in dendrites particularly along microtubules ${ }^{4.30}$ and in axons $s^{4,26}$, although others have failed to demonstrate the latter distribution $^{11,28}$.

But the neuron has a high degree of regional specialization. Axons, particularly myelinated axons, show a heterogeneous distribution of structural and functional macromolecules related to particular functions at limited sites in the axon. In order to help define which of the regulatory processes, demonstrated in vitro as depending on calmodulin, are relevant in nerve, the distribution of calmodulin must be compared to the known distribution of those proteins which might be regulated by calmodulin.

In this paper we report the immunohistochemical distribution of calmodulin on axons of the rat sciatic

Correspondence: M. Mata, Neurology Dept. (127), 2215 Fuller Road, Ann Arbor, MI 48105, U.S.A. 


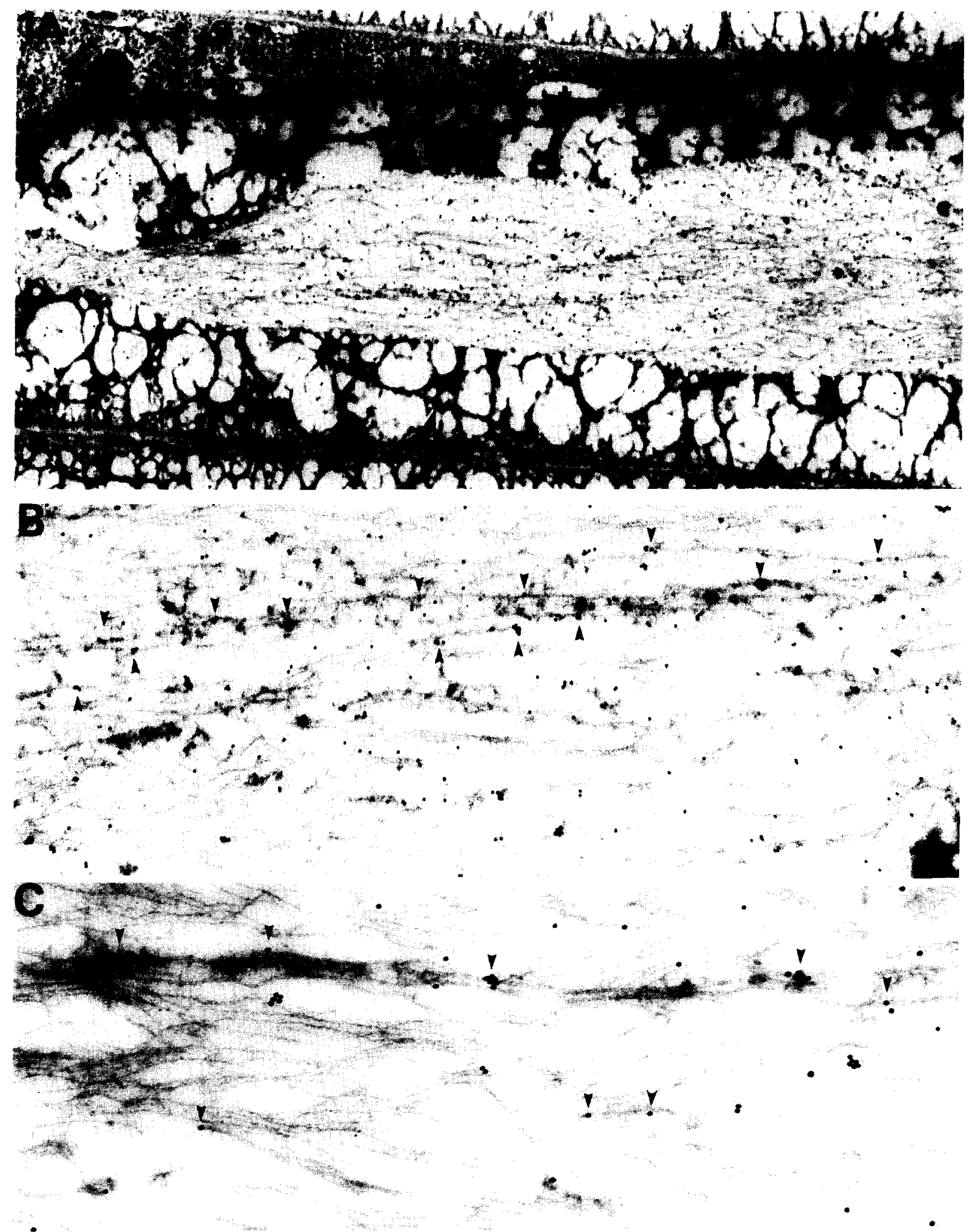

Fig. 1. Longitudinal section of sciatic nerve demonstrates calmodulin immunoreactivity in the nucleus and cytoplasm of the Schwann cell (s), and along the Schmidt-Lanterman cleft (arrows). Calmodulin immunoreactivity in the axoplasm is diffusely distributed, preferentially along microtubules (arrowheads). A, $\times 10,920 ; \mathrm{B}, \times 28,560 ; \mathrm{C}, \times 50,400$. 
nerve as defined at the ultrastructural level, and interpret that data in light of the known regional specialization of nerve.

\section{MATERIALS AND METHODS}

All studies were performed on Sprague-Dawley
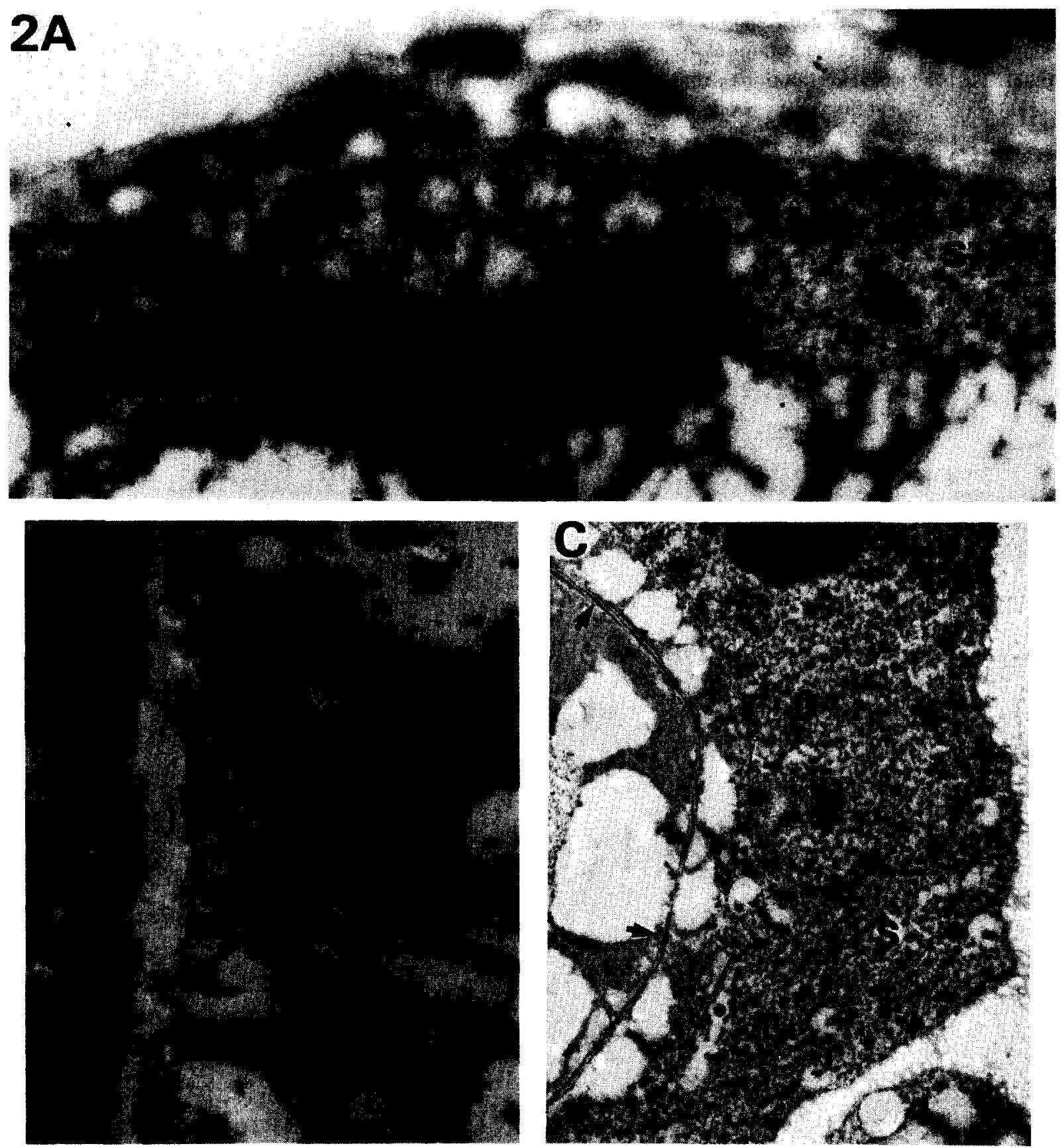

Fig. 2. A and B: high-power view of Schmidt-Lanterman clefts show immunoreactivity predominantly in the cytoplasm of the cleft (arrows). In A, the Schmidt-Lanterman cleft can be seen opening into the Schwann cell cytoplasm (s). C: calmodulin immunoreactivity is seen in the nucleus and cytoplasm of a Schwann cell (s), and in the Schmidt-Lanterman cleft (arrows). A, $\times 37,800 ; \mathrm{B}, \times 29,400 ; \mathrm{C}$, $\times 19,320$. 
rats weighing $200-250 \mathrm{~g}$. The animals were perfused through the heart with $100 \mathrm{mM}$ phosphate buffer followed by $0.5 \%$ glutaraldehyde, $4 \%$ paraformaldehyde in the same buffer. Blocks of the rat sciatic nerve were placed in the same fixative for an additional $2 \mathrm{~h}$ and then embedded in LR white. Ultrathin sections were exposed to affinity-purified sheep anticalmodulin (raised against calmodulin from bovine brain, Polysciences) in dilutions from 1:1000 to 1:2000 for $4-72 \mathrm{~h}$, followed by rabbit anti-goat $\operatorname{IgG}$ bound to $15 \mathrm{mM}$ colloidal gold (E-Y Laboratories), and the grids were stained for $20 \mathrm{~min}$ with uranyl acetate and examined in a JEOL 100 S electron microscope.

Controls for the histochemical reaction included deletion of the anti-calmodulin antibody, or pre-absorption of the anti-calmodulin antibody independently with purified calmodulin from bovine brain (Sigma) and from bovine testis (Polysciences).
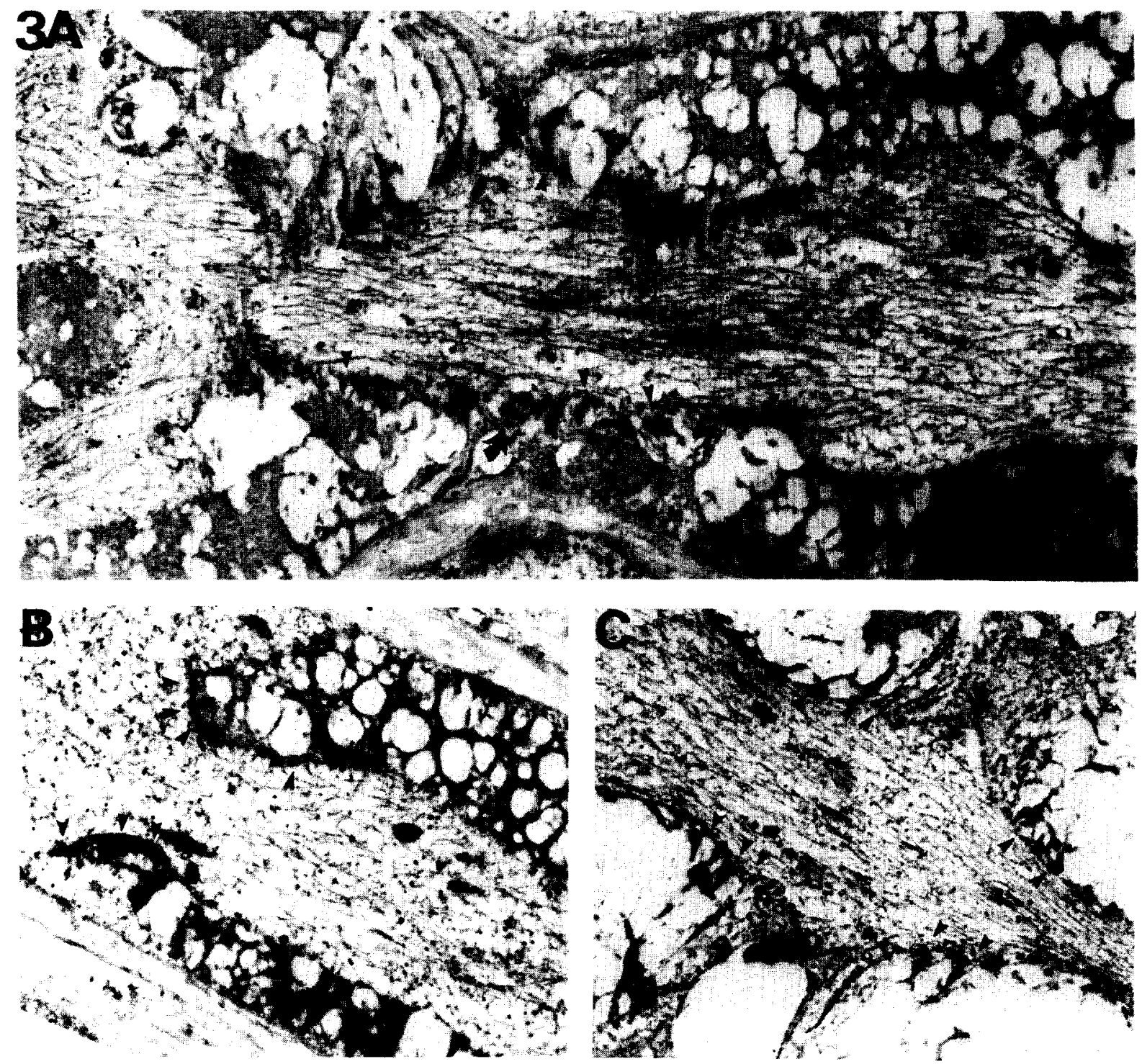

Fig. 3. At the node of Ranvier immunogold deposition is seen particularly in the paranodal region (arrowheads) while the nodal axolemma shows little or no immunoreactivity (arrows). A, $\times 16,128 ; \mathrm{B}, \times 18,480 ; \mathrm{C}, \times 14,700$. 


\section{RESULTS}

Within axons, calmodulin immunoreactivity was seen diffusely throughout the axoplasmic matrix. The distribution was homogeneous and did not demonstrate the gradients which we have previously found for the distribution of calcium in myelinated axons. Immunogold labeling was deposited preferentially along microtubules and occasionally the axolemma (Fig. 1).

In Schwann cells, calmodulin immunoreactivity appeared in the cytoplasm and in the nucleus (Figs. $1 \mathrm{~A}, 2 \mathrm{C}$ ). There was no immunoreactivity along the membranes forming compact myelin, but we found consistently intense immunogold deposition within the Schmidt-Lanterman clefts (Fig. 2). This distribution appeared within the cytoplasm of the cleft, not in the membranes.

At the nodes of Ranvier calmodulin immunoreactivity was seen predominantly in the paranodal region, along the junction of the paranodal axolemma and the paranodal Schwann cell membrane (Fig. 3). With the resolution of this technique it is not possible to determine whether that immunoreactivity is axolemmal or within the paranodal loops. At greater distances from the center of the axon, however, it was clear that the immunoreactivity was found in the paranodal loops of myelin formed by the Schwann cell (Fig. 4).

Although immunolabeling is not strictly quantita-
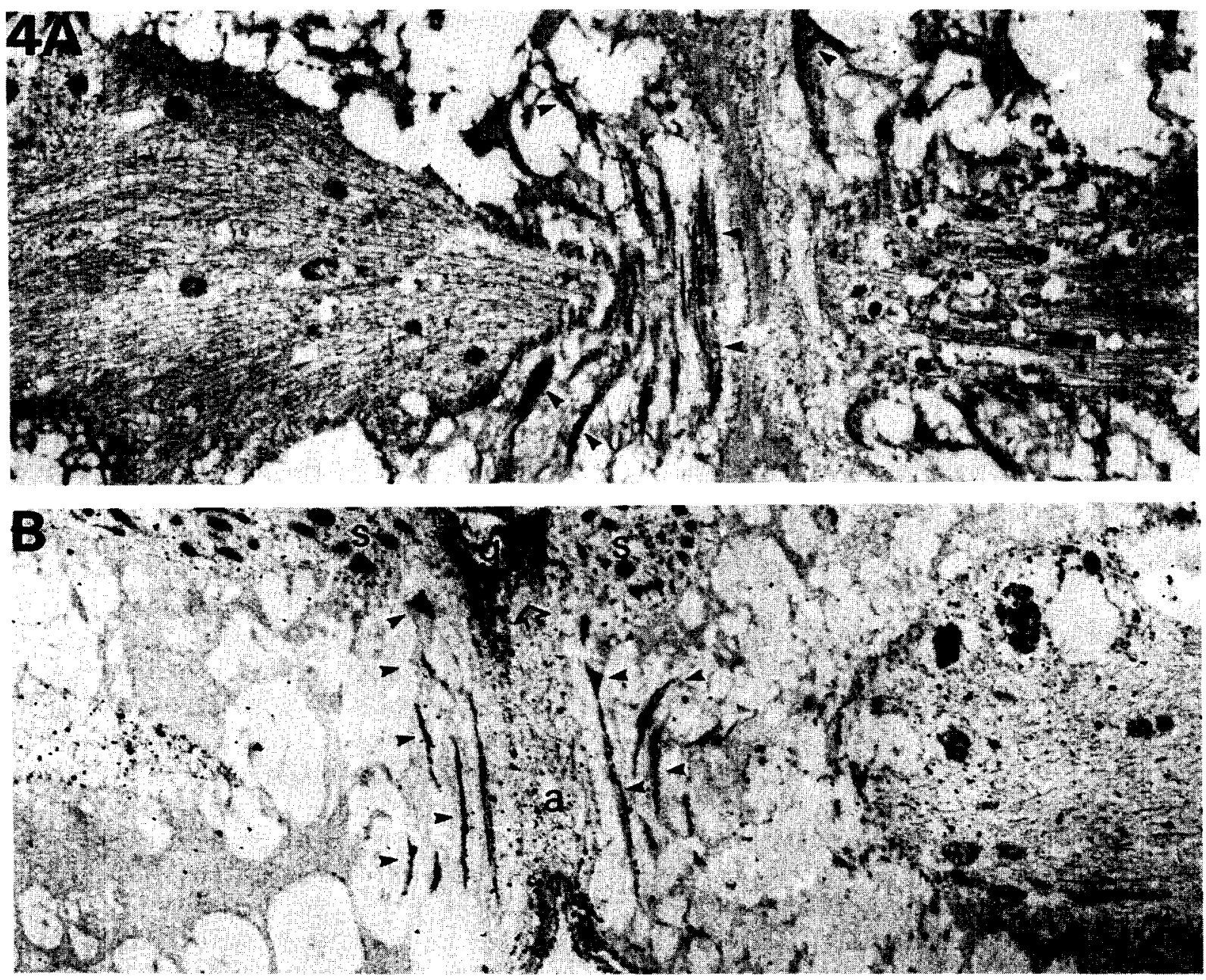

Fig. 4. Calmodulin immunoreactivity is seen in the paranodal loops of myelin (arrowheads) about the node of Ranvier in these longitudinal sections cut away from the center of the axon. Note the absence of immunoreactivity along the axonal membrane at the node (open arrow). s, Schwann cell; g, ground substance. A, ×17,640; B, ×14,280. 

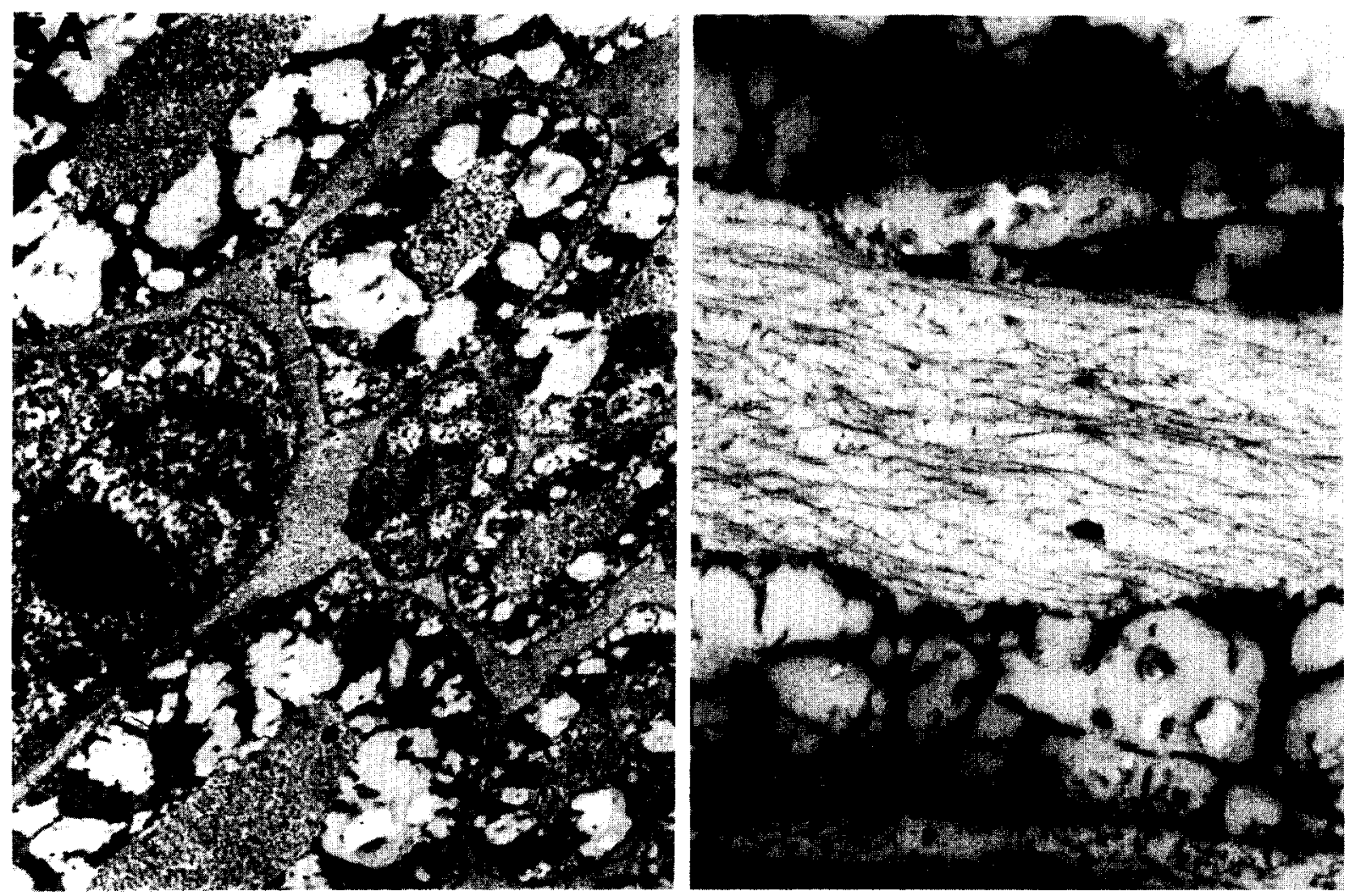

Fig. 5. A: control using preabsorption of the anti-calmodulin antibody $(1: 2000)$ with calmodulin $(0.4 \mathrm{mg} / \mathrm{ml}) . \times 9720 . \mathrm{B}: \mathrm{control}$ using deletion of the primary antibody. Subsequent steps were carried out as described. $\times 14,580$.

tive, the amount of labeling in the Schmidt-Lanterman clefts and in the paranodal region was much greater than that in other regions, and appeared earlier at higher dilutions of the primary antibody.

Control sections did not demonstrate immunoreactivity (Fig. 5).

\section{DISCUSSION}

This is the first study of CaM immunoreactivity in the peripheral nervous system. We found calmodulin immunoreactivity within the axoplasm, confirming the distribution which has previously been described in the CNS (hippocampus, cerebellum ${ }^{4}$, and cerebrum $^{26}$ ) and in rat superior cervical ganglion ${ }^{22}$. We found immunogold deposition within the axons preferentially along microtubules. This localization is in agreement with the biochemical demonstration that newly synthesized calmodulin is transported down axons with the slow transport of microtubules ${ }^{2,16}$.
There is biochemical evidence that calmodulin is involved in the regulation of microtubule polymerization by calcium ${ }^{1}$. There is conflicting evidence regarding whether calmodulin binds directly to tubulin ${ }^{7}$ or to microtubule-associated proteins ${ }^{12.24}$. A calcium/calmodulin-dependent kinase (CaM kinase II) co-purifies with tubulin ${ }^{10.27}$, and has been proposed to regulate microtubule assembly and microfilament-neurofilament interactions through phosphorylation of microtubule-associated protein ${ }^{28}$. Monoclonal antibodies raised against calmodulin-dependent kinase appear to label microtubules ${ }^{28}$ though previous reports with a different monoclonal antibody showed only faint axonal immunoreactivity ${ }^{18}$.

In addition to its role in regulating assembly of the cytoskeleton, indirect evidence implicates calmodulin in rapid axonal transport which takes place along microtubules. Calmodulin antagonists inhibit both anterograde and retrograde axonal transport in concentrations that do not disrupt microtubules ${ }^{6,11}$. The 
direct localization of calmodulin along microtubules in the current study supports such a role for calmodulin.

It should be noted that we did not see gradients in calmodulin distribution that we have previously seen in calcium distribution ${ }^{14}$. This suggests that those calcium gradients are not a result of gradients in the distribution of the binding proteins.

In the PNS we found that all Schwann cells stained unequivocally in their cytoplasm and nuclei. This is in accord with the virtually universal distribution of calmodulin, though in contrast to the CNS immunoreactivity in glia which has been described as weak ${ }^{26}$ or found in only some glial cells ${ }^{4}$. We found particularly intense immunoreactivity in Schmidt-Lanterman clefts. The function of these structures is not known, though they are known to contain microtubules. In our oxalate-pyroantimonate studies of calcium distribution we found a similar distribution of calcium precipitate within the Schmidt-Lanterman clefts ${ }^{14}$. The distribution of calmodulin and calcium suggests that a calcium/calmodulin-mediated function may be carried out by these structures.

We also found calmodulin in the paranodal loops at the node of Ranvier. We have recently demon-

\section{REFERENCES}

1 Bender, P.K. and Rebhun, L.I., The calcium sensitivity of MAP-2 and tau microtubules in the presence of calcium, Ann. N.Y. Acad. Sci., 466 (1986) 392-409.

2 Brady, S., Tytell, M., Heriot, K. and Lasek, R.J., Axonal transport of calmodulin; a physiological approach to identification of long term association between proteins, J. Cell Biol., 89 (1981) 607-614.

3 Brostrom, C.O., Brostrom, M.A. and Wolff, D.J., Calcium-dependent adenylate cyclase from rat cerebral cortex, J. Biol. Chem., 252 (1977) 5677-5685.

4 Carceres, A., Bender, P., Snavely, L., Rebhun, L. and Steward, O., Distribution and subcellular localization of calmodulin in adult and developing brain tissue, Neuroscience, 10 (1983) 449-461.

5 Cheung, W.Y., Cyclic 3',5'-nucleotide phosphodiesterase: demonstration of an activator, Biochem. Biophys. Res. Commun., 38 (1970) 533-538.

6 Edstrom, A., Ekstrom, P., Kanje, M. and Sjoberg, J., The use of the regenerating frog sciatic nerve for pharmacological studies of orthograde and retrograde axonal transport, Brain Research, 401 (1987) 34-42.

7 Ekstrom, P. and Kanje, M., Calmodulin-binding proteins within the slow phase of axonal transport in the rabbit vagus nerve, J. Neurochem., 49 (1987) 146-151.

8 Gopinath, R.M. and Vincenzi, F.F., Phosphodiesterase protein activator of $\left(\mathrm{Ca}^{2+}+\mathrm{Mg}^{2+}\right)$ ATPase, Biochem. Bio. strated that Ca-ATPase activity in myelinated fibers (determined by enzyme cytochemistry) has a similar distribution with a particularly dense reaction in paranodal loops ${ }^{15}$. The erythrocyte has a Ca-ATPase on its membrane which is regulated by calmodulin ${ }^{8}$ and synaptic plasma membranes isolated from mammalian brain also contain a $\mathrm{Ca}, \mathrm{Mg}$-ATPase with a calmodulin-regulatable component ${ }^{25}$. The co-localization at paranodal loops in peripheral myelinated fibers of calcium ${ }^{14}$, Ca-ATPase activity ${ }^{15}$, and CaM immunoreactivity strongly suggests that at that site small increases in cytosolic calcium may serve to activate the calcium pump through calmodulin.

\section{ACKNOWLEDGEMENTS}

This work supported by VA Merit Review Grants to M.M. and D.J.F., and a grant from the Michigan Diabetes Research and Training Center to D.J.F. We wish to thank Melissa Tuck for technical assistance in electron microscopy, and Anna Boda and Glen Gersten for assisting in photography. A preliminary communication of these results was presented at the annual meeting of the American Academy of Neurology in Cincinnati in April, 1988.

phys. Res. Commun., 77 (1977) 1203-1209.

9 Hinds, T.R., Larsen, F.L. and Vincenzi, F.F., Plasma membrane $\mathrm{Ca}^{2+}$ transport; stimulation by soluble proteins, Biochem. Biophys. Res. Commun., 81 (1978) 455-461.

10 Larson, R., Goldenring, J.R., Vallano, M.L. et al., Identification of endogenous calmodulin-dependent kinase and calmodulin-binding proteins in cold-stable microtubule preparations from rat brain, $J$. Neurochem., 44 (1985) $1566-1574$.

11 Lavoie, P.-A. and Tiberi, M., Inhibition of fast axonal transport in bullfrog nerves by dibenzazepine and dibenzocycloheptadine calmodulin inhibitors, J. Neurobiol., 17 (1986) 681-695.

12 Lee, Y.C. and Wolff, J., Calmodulin binds to both microtubule associated protein 2 and tau protein, J. Biol. Chem., 259 (1984) 1226-1230.

13 Lin, C.T., Dedman, J.R., Brinkley, B.R. and Means, A.R., Localization of calmodulin in rat cerebellum by immunoelectron microscopy, J. Cell Biol., 85 (1986) 473-480.

14 Mata, M., Staple, J. and Fink, D.J., Ultrastructural localization of calcium within neurons; and oxalate pyroantimonate study, Histochemistry, 87 (1987) 339-349.

15 Mata, M., Staple, J. and Fink, D.J., Cytochemical localization of $\mathrm{Ca}^{2+}$-ATPase activity in periphera nerve, Brain $R e$ search, in press.

16 McQuarrie, I., Brady, S. and Lasek, R.J., Diversity in the axonal transport of structural proteins; major differences between optic and spinal axons in the rat, J. Neurosci., 6 
(1986) 1593-1605.

17 Means, A.R., Tash, J.S. and Chafouleas, J.G., Physiological implications of the presence, distribution, and regula. tion of calmodulin in eukaryotic cells, Physiol. Rev., 62 (1982) 1-39.

18 Ouimet, C.C., McGuinness, T.L. and Greengard, P., Immunocytochemical localization of calcium/calmodulin-dependent protein kinase II in rat brain, Proc. Natl. Acad. Sci. U.S.A., 81 (1984) 5604-5608.

19 Rebhun, L.I., Jemiolo, D., Keller, T., Burgess, W. and Kretsinger, R., Calcium-calmodulin and control of assembly of brain and spindle microtubules. In M. Debrabander and J. DeMey (Eds.), Microtubules and Microtubule Inhibitors, Vol. 3, Elsevier, Amsterdam, 1980, pp. 243-252.

20 Scholey, J.M., Taylor, K.A. and Kendrick-Jones, J., Regulation of non-muscle myosin assembly by calmodulin-dependent light chain kinase, Nature (Lond.), 287 (1980) 233-235.

21 Seto-Ohshima, A., Kitajima, S., Sano, M., Karo, K. and Mizutani, A., Immunohistochemical localization of calmodulin in mouse brain, Histochemistry, 79 (1983) 251-257.

22 Seto-Ohshima, A., Sano, M., Kitajima, S., Kawamura, N., Yamazaki, Y. and Nagato, Y., The effect of axotomy and denervation on calmodulin content in the superior cervical sympathetic ganglion of the rat, Brain Research, 410 (1987) 292-298.

23 Smoake, J.A. and Solomon, S.S., Subcellular shifts in cyclic AMP phosphodiesterase and its calcium dependent regulator in liver. Role of diabetes, Biochem. Biophys. Res. Commun., 94 (1980) 424-430.

24 Sobue, K., Fujita, M., Muramato, Y. and Kakiuchi, S.,
The calmodulin binding protein in microtubules is tau factor, FEBS Lett., 132 (1981) 137-140.

25 Sobue, K., Ichida, S., Yoshida, H., Yamazaki, R. and Kakiuchi, S., Occurrence of a $\mathrm{Ca}^{2+}$ and modulator protein-activatable ATPase in the synaptic plasma membranes of brain, FEBS Lett., 99 (1979) 100-201.

26 Tabuchi, K., Ohnishi, R., Nishimoto, A., Osobe, T. and Okuyama, T., Reverse cellular distribution of calmodulin to S-100 protein in primate brain, Brain Research, 298 (1984) 353-357.

27 Vallano, M.L., Goldenring, J.R., Buckholz, T.M. et al., Separation of endogenous calmodulin- and cAMP-dependent kinases from microtubule preparations, Proc. Natl. Acad. Sci. U.S.A., 82 (1985) 3202-3206.

28 Vallano, M.L., Goldenring, J.R., Lasher, R.S. and DeLorenzo, R.J., Association of calcium/calmodulin-dependent kinase with cytoskeletal preparations: phosphorylation of tubulin, neurofilament, and microtubule-associated proteins, Ann. N.Y. Acad. Sci., 466 (1986) 357-374.

29 Wolff, D.J., Ross, J.M., Thompson, P.N., Brostrom, M.A. and Brostrom, C.O., Interaction of calmodulin with histones; alterations of histone dephosphorylation, J. Biol. Chem., 256 (1981) 1846-1860.

30 Wood, J.G., Wallace, R.W., Whitaker, J.N. and Cheung, W.Y., Immunocytochemical localization of calmodulin and a heat-labile calmodulin-binding protein (CAM-BP80) in basal ganglia of mouse brain, J. Cell Biol., 84 (1980) 66-76.

31 Yamaki, T. and Hidaka, H., $\mathrm{Ca}^{2+}$ independent stimulation of cyclic GMP-dependent stimulation of cyclic AMP-dependent protein kinase by calmodulin, Biochem. Biophys. Res. Commun., 94 (1980) 727-733. 